Meta

Journal des traducteurs

Translators' Journal

\title{
Le statut de la formation des mots savants en français et en anglais
}

\section{Wiecher Zwanenburg}

Volume 32, numéro 3, septembre 1987

La fertilisation terminologique dans les langues romanes

URI : https://id.erudit.org/iderudit/004600ar

DOI : https://doi.org/10.7202/004600ar

Aller au sommaire du numéro

Éditeur(s)

Les Presses de l'Université de Montréal

ISSN

0026-0452 (imprimé)

1492-1421 (numérique)

Découvrir la revue

Citer cet article

Zwanenburg, W. (1987). Le statut de la formation des mots savants en français et en anglais. Meta, 32(3), 223-229. https://doi.org/10.7202/004600ar d'utilisation que vous pouvez consulter en ligne. 


\title{
LE STATUT DE LA FORMATION DES MOTS SAVANTS EN FRANÇAIS ET EN ANGLAIS
}

\author{
WIECHER ZWANENBURG \\ Université d'Utrecht, Pays-Bas
}

Dans les langues du monde, la formation des mots peut se réaliser en principe sous deux formes. La dérivation permet de former des mots en combinant un radical et un affixe, et la composition permet de former des mots en combinant plusieurs radicaux. Une langue peut ne présenter qu'une de ces deux formations ou même aucune. Mais les langues de culture occidentales possèdent toutes les deux. Et à cet égard-là, le français et l'anglais ne se distinguent donc pas l'un de l'autre.

En outre, les langues de culture occidentales se ressemblent entre elles par le fait qu'elles présentent, à côté de la dérivation et de la composition ordinaires, une dérivation et une composition savante. Comparez (1)(a) et (1)(b) pour des exemples français et anglais respectivement.

(1) (a) français
dérivation :
composition :
aboiement
stimulation
timbre-poste
démo-crate, agri-culture

non savante :

savante :

(b) anglais

$\begin{array}{lll}\text { dérivation : } & \text { barking } & \text { stimulation } \\ \text { composition : } & \text { postage stamp } & \text { demo-crat, agri-culture }\end{array}$

Les exemples démocrate et agriculture, democrat et agriculture sont là pour montrer que la composition savante peut se faire en principe à l'aide d'éléments grecs et latins respectivement. En dérivation, il s'agit en général d'affixes savants d'origine latine, dont certains remontent à leur tour au grec. Les principaux suffixes latins empruntés au grec sont -ique, -iser, -isme et -iste en français et les suffixes correspondants en anglais.

Ce que les exemples de (1) montrent surtout, c'est que très souvent les dérivés et composés savants se correspondent d'une langue moderne à l'autre. Cela tient partiellement au fait qu'il s'agit de mots empruntés directement ou indirectement aux deux langues classiques. Mais souvent aussi il s'agit de mots dérivés et composés non empruntés, mais formés d'après le modèle grec ou latin. Un dérivé savant qui ne saurait être un emprunt est par exemple :

(2) chiracquisation

Le français et l'anglais ne se ressemblent donc pas seulement par la distinction qu'ils font entre dérivation et composition. Mais ils se ressemblent en outre, de façon plus frappante et en même temps plus superficielle, par le fait qu'ils présentent une dérivation et une composition savante qui font souvent usage des mêmes éléments empruntés au grec et au latin.

Ce que je voudrais faire ici, c'est attirer l'attention sur deux différences importantes entre la formation des mots savants en français et en anglais. Ces différences font que le statut de la formation des mots savants en français est autre qu'en anglais, malgré les ressemblances superficielles. 
J'examinerai d'abord les rapports hiérarchiques entre dérivation et composition dans les deux langues. J'entends par là la façon dont dérivation et composition peuvent être combinées à des niveaux différents dans la structure d'un mot. Ensuite je discuterai les rapports formels entre formation savante et non savante. Cela concerne les ressemblances de forme qu'on peut constater entre certains éléments non savants et savants, comme -aison et -ation, -eté et -ité dans (3).

(3) pend-aison / sépar-ation,

dur-eté / dign-ité

1. Considérons d'abord les rapports hiérarchiques entre formation savante et non savante en français et en anglais.

Prenons notre point de départ dans Pichon (1942: 47-49). Pichon a observé qu'en français un suffixe savant ne peut s'attacher à un radical savant aussi bien qu'à un radical non savant. Voir les exemples de (4), avec-ation savant et -aison non savant, germinsavant et germ- non savant.

(4) (a) germin-ation / *germ-ation

(b) germin-aison / germ-aison

Cela implique un rapport hiérarchique entre dérivation savante et non savante. C'est qu'on peut attacher un suffixe savant à un radical savant et ensuite ajouter un suffixe non savant, témoin perfectionnement dans (5)(a). Mais l'inverse n'est pas possible, témoin l'agrammaticalité de *ballottation au lieu de ballottement dans (5)(b).

(5) (a) [perfect-ionn]ement

(b) *[ball-ott]ation

Cela veut dire qu'il y a deux couches d'affixes de dérivation. Il y a une couche inférieure d'affixes savants et une couche supérieure d'affixes non savants.

Cette observation de Pichon a passé quasi inaperçue. Mais plus tard on a observé le même phénomène pour l'anglais, comparez Selkirk (1982: 77-119), qui se base surtout sur Chomsky-Halle (1968) et Siegel (1974). Voyez les exemples de (6), où N et $\mathrm{A}$ indiquent nom et adjectif et où les lignes courbées entourent les éléments savants

(6)

(a)

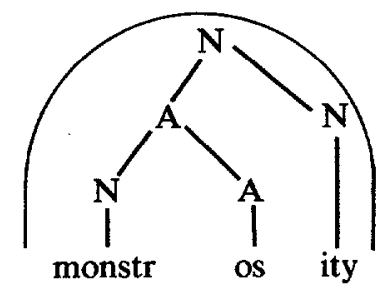

(c)

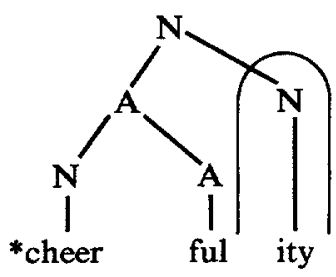

(b)

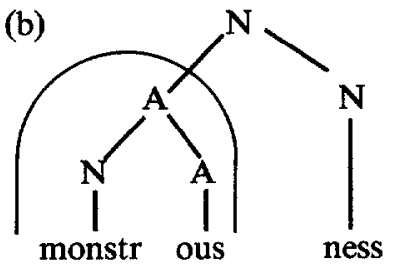

(d)

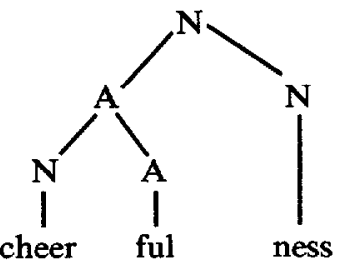

Pour l'anglais il est plus facile d'observer ce phénomène, parce que dans cette langue on rencontre souvent des suffixes non savants après des suffixes savants. En français il y a une certaine tendance à combiner suffixes savants ou non savants entre eux, et des exemples comme ceux de (5) y sont donc moins abondants. Mais cela n'empêche que les 
combinaisons de suffixes qu'on y rencontre respectent la même contrainte. Et de toute façon les combinaisons de racine plus suffixe comme dans (4) illustrent le phénomène abondamment.

On peut faire des observations comparables pour la préfixation.

Nous pouvons donc conclure qu'il y a deux couches d'affixations en français comme en anglais, une couche savante inférieure et une couche non savante supérieure. Comme l'illustrent tous les exemples de (4) à (6), il s'agit d'une distinction formelle. Au point de vue morphologique, le suffixe savant -ation et les non savants -aison et -ement ont la même fonction en français : former un nom d'action sur la base d'un radical verbal. Et il en est de même pour le suffixe savant -ity et le non savant -ness en anglais : ils forment un nom abstrait sur la base d'un radical adjectival.

Venons-en à la composition. Nyrop (1908: 39-40) fait remarquer qu'il est rare qu'en français un mot composé serve de radical pour former un mot dérivé. Il cite des exemples exceptionnels comme ceux de (7).

(7) basbleuisme, centre-droitier, chatnoiresque ; beaupérisme, bonbockeur, dixseptième-siècliste

Il s'agit dans la plupart de ces cas de créations ad hoc. Il est vrai qu'on rencontre de rares exemples de ce genre dans le lexique ordinaire du français, comme ceux de (8).

(8) extrême-oriental, grand-ducal, moyenâgeux

Cela me mènerait trop loin dans ce cadre de montrer pourquoi de tels cas exceptionnels se rencontrent dans le lexique ordinaire et dans quelles conditions exactement. Voir cependant la fin de ce paragraphe et, pour une discussion plus détaillée, Zwanenburg (1986).

De toute façon la règle générale est celle formulée par Nyrop. Et cela veut dire qu'en français la composition constitue une couche supérieure à la dérivation non savante.

Il en est autrement en anglais. Comparez les exemples de (9).

(9) (a) [neighborhood] [planning], [brownstone] [neigborhood], [programs] [coordinator]

(b) [stuck-up]ness, [runaway]hood, [pickup]ful

On constate que dans (9)(a) les suffixes - hood et -or se rencontrent à l'intérieur d'un mot composé. Et dans (9)(b) on trouve l'inverse : les suffixes -ness, -hood et -ful y sont combinés avec un mot composé. Dans de tels cas on a toujours affaire à des suffixes non savants. Et on peut, par conséquent, conclure qu'en anglais la dérivation non savante et la composition constituent une seule couche hiérarchique.

Mais tout cela ne concerne que la composition non savante. Ce qui a été moins remarqué, notamment pour l'anglais, c'est qu'il y a aussi une composition savante.

Voici d'abord quelques exemples français : ceux de gauche d'origine grecque, ceux de droite d'origine latine ( $V$ indique le verbe) :

(10)
(a) noms :
N N : aérodrome
agriculture
A $\mathbf{N}$ : hiéroglyphe
(b) adjectifs :
$\mathrm{N} N$ : anthropomorphe
A $\mathbf{N}$ : hétérodoxe
rectangle
cunéiforme
magnanime
$\mathrm{V} N$ : pathogène
centrifuge

Il n'est guère nécessaire de donner des exemples anglais de composition savante, puisque les deux langues sont tout à fait comparables à cet égard. Très souvent, elles se servent des mêmes éléments. Pour le constater, il suffit de comparer les exemples français de (11)(a) avec les exemples anglais de (11)(b) : 
(11) (a) automobile, érithrocyte, ethnocentr(ique), francophile, microscope, nullipare, polygone

(b) automobile, erythrocyte, ethnocentr(ic), francophile, microscope, nullipar(ous), polygon

Examinons maintenant la place de la composition savante dans la hiérarchie de la formation des mots dans les deux langues. Mais disons d'abord quelque chose sur la différence entre composition et dérivation savante. Cela est nécessaire, parce qu'on décrit parfois certains éléments de composition savante comme des affixes. C'est le cas, par exemple, de -phile dans francophile ou de -graphe et -logue dans géographe et géologue.

Cela est compréhensible, puisque la plupart des éléments de composition savante ne se rencontrent pas à l'état isolé comme mots. Et c'est une particularité qu'ils partagent avec les affixes, bien sûr. Mais à y regarder de près, il n'est pas possible de considérer ces éléments de composition savante comme des affixes. Tout d'abord cela signifierait que les mots cités dans (10) et (11) ne comporteraient que des affixes. Et cela constitue une contradiction dans les termes : un vrai affixe demande un radical avec lequel il puisse se combiner. En outre, certains des éléments de composition savante s'emploient tantôt comme premier élément, tantôt comme second, témoin (12) :

(12) philo-logie / franco-phile

Et les affixes sont toujours soit préfixes soit suffixes, soit, dans d'autres langues, infixes.

Il y a donc tout lieu de distinguer la composition savante d'avec la dérivation. II faut seulement tenir compte du fait que la distinction est partiellement déterminée par le rapport de la formation savante avec le grec et le latin, où l'on rencontre les éléments de composition savante à l'état isolé comme mots. Cela n'est pas pour nous étonner, puisque la formation savante, même là où elle se manifeste dans des mots non empruntés mais créés en français ou en anglais, suit toujours de près les modèles grecs et latins. Voir Zwanenburg (1983). Il y a, en d'autres termes, une symbiose intéressante entre le français ou l'anglais et les langues classiques dans le domaine de la formation des mots savants.

Une fois la distinction faite entre composition et dérivation savante, on doit se demander quelle est la place de la composition savante dans la hiérarchie de la formation des mots en français et en anglais. Or, on constate que la composition savante est toujours enchâssée dans la dérivation, savante ou non savante, et jamais l'inverse. Considérez, pour le constater, les exemples français de (13) :

(13) (a) [acro-bat]ique, [aristo-crat]ie, [auto-mat]is(er)

(b) [arti-fic]ier, [belli-gér]ant, [chrono-métr]eur

Les exemples de (13)(a) ont un suffixe savant, ceux de (13)(b) un suffixe non savant. Il serait facile de donner des exemples comparables pour l'anglais.

La conclusion qu'on peut en tirer, c'est que dans les deux langues la composition savante constitue une couche à part, inférieure à la dérivation savante.

On peut résumer ce qui a été dit jusqu'ici dans les deux tableaux suivants, présentant les différentes couches de la formation des mots en français et en anglais respectivement, en commençant par la couche inférieure :

(14) (a) français composition savante dérivation savante dérivation non savante composition non savante (b) anglais : composition savante dérivation savante dérivation non savante composition non savante

Cela veut dire que la place de la composition savante n'est pas différente dans les deux langues, en tout cas à première vue. Mais si l'on y regarde de plus près, il faut constater qu'il y a quand même une différence fondamentale. En français, et en français seu- 
lement, la composition savante est la seule forme de composition qui puisse être enchâssée dans la dérivation. En anglais, par contre, tant les composés savants que les composés non savants peuvent servir de base à des mots dérivés.

Il est possible que cette différence provienne d'une différence structurale entre la composition non savante française et l'anglaise. Considérez les exemples suivants de composition savante et non savante dans les deux langues :

(15) (a) français :

francophile timbre-poste (b) anglais :

francophile

postage stamp

Les éléments en italiques dans (15) sont les déterminés, les éléments centraux. Les autres sont les déterminants, les éléments complémentaires. Or, on constate que de façon générale la composition savante a le déterminé à droite dans les deux langues. Mais la composition non savante a le déterminé à gauche en français, comme dans la syntaxe française, et à droite en anglais, comme souvent dans la syntaxe anglaise. En d'autres termes, la composition non savante française se distingue par le fait qu'elle a le déterminé à gauche.

Considérez maintenant les exemples agrammaticaux français de (16)(a) avec les exemples grammaticaux anglais de (16)(b) :

(16) (a) *[amour-propr]eux, *[eau-bénit]ique

(b) [blue-stocking]ish, [strong-mind]ed

En anglais, le déterminé du composé est à droite, et ce déterminé est par définition de la même catégorie que le composé, celle du nom. Cela fait que le suffixe qu'on ajoute à la droite du composé s'ajoute immédiatement à la droite du déterminé de la même catégorie, et cela donne une succession acceptable : -ish et -ed demandent un radical nominal et la succession immédiate stocking-ish, mind-ed obéit à cette régularité. Mais, dans les exemples français, avec le déterminé à gauche, l'adjonction d'un suffixe au composé n'a pas cet effet : -eux et -ique demandent aussi un radical nominal, mais la succession immédiate *propr-eux, *bénit-ique viole cette régularité.

Il est donc possible que le non-enchâssement de la composition non savante dans la dérivation en français soit dû au fait que le déterminé est normalement à gauche. Cela s'accorderait avec la présence de certains composés non savants enchâssés exceptionnellement dans la dérivation, comme ceux de (8) : dans ces cas le composé a exceptionnellement son déterminé à droite.

Quoi qu'il en soit, la composition savante française est la seule forme de composition dans cette langue qui puisse normalement être enchâssée dans la dérivation. Et c'est là un point important pour son emploi à des fins terminologiques. C'est qu'elle permet ainsi ce que ne permet pas la dérivation non savante : elle permet de développer un système terminologique plus étendu, comme dans (17).

(17) monarque, monarchie, monarchique, monarchisme, monarchiste

2. Examinons maintenant les rapports formels entre formation savante et non savante en français et en anglais.

Il est connu que le français possède pas mal de doublets, c'est-à-dire de mots se présentant sous une forme non savante et savante, notamment latine, en général avec une différence de sens :

(18) chétif / captif, étrangler / stranguler, frêle / fragile

Cela tient évidemment à l'histoire du français, dont le lexique est pour la plus grande partie d'origine latine et a été enrichi en outre depuis le Moyen Âge par des emprunts au latin. Cela explique qu'il s'agit de cas incidentels, dus aux hasards du développement de 
la culture qui a amené l'emprunt de certains mots dont l'équivalent non savant existait déjà dans la langue.

Mais c'est au niveau des morphèmes, racines et affixes, que le phénomène se manifeste de façon beaucoup plus systématique. Dell-Selkirk (1978) ont observé que beaucoup de racines ont une forme non savante quand elles sont employées comme mots ou devant un suffixe non savant, et une forme savante devant un suffixe savant :

(19) (a) mer

fleur

a-merr-ir mar-in

fleur-ir flor-al

(b) seul

seul-ement

(c) école

lettré

(d) estomac

écol-ier

sol-itude

scol-aire

Les parties en italiques indiquent certaines correspondances formelles entre formes non savantes et savantes, qui se retrouvent régulièrement dans de telles paires de formes. Ainsi le è et le $e u$ sont régulièrement postériorisés en $a$ et $o$ dans les formes savantes, comme dans mer/marin et fleur/floral.

Ces mêmes alternances se retrouvent dans les suffixes savants, employés à la fin du mot et devant un ou plusieurs autres suffixes respectivement :
(20) rationn-el
nerv-eux
ration- $a$ l-is-er
nerv-osité

Finalement, le français connaît un certain nombre de paires d'affixes non savants et savants qui présentent les mêmes alternances :
(21) livr-aison migr-ation
chapel-ain rom-an
duch-é épiscop-at
charn-el caud- $a$ l

On constate des faits comparables pour la composition savante, mais ici ils sont un peu moins abondants. C'est que la composition savante est pour une très grande partie d'origine grecque, où le phénomène ne saurait se manifester, à l'encontre de la dérivation savante, qui est surtout d'origine latine. Comparez cependant les exemples suivants, où dérivation et composition savante se correspondent au point de vue formel :

(22) père fleur

patr-ie patri-arc(at)

flor-al

$$
\text { flori-lège }
$$

Ainsi nous constatons qu'il y a un rapport formel assez étroit entre la formation des mots savants et non savants en français. Ce rapport se manifeste dans des correspondances phonologiques souvent très régulières et très répandues dans le lexique.

Il n'en est rien dans le lexique anglais. Dans cette langue, des alternances comme celles discutées ci-dessus pour le français se manifestent en général à l'intérieur du lexique savant. Comparez pour cela Chomsky-Halle (1968). Mais une racine ou un affixe savant n'a en général aucun rapport formel avec la racine ou l'affixe non savant qui lui correspond. Comparez :
(23) sea alone
mar-ine sol-itude

Et pour autant qu'il y a des racines non savantes d'origine romane, leur rapport formel avec des racines savantes est beaucoup moins étroit et régulier en français qu'en anglais
(24) clear flower clar-ify flor-al

Cela nous permet de conclure qu'en français la formation des mots savants, notamment ceux d'origine latine, est liée beaucoup plus étroitement à la formation non savante au point de vue formel qu'en anglais. Il me semble permis de conclure que la for- 
mation des mots dans son ensemble constitue ainsi un ensemble beaucoup plus homogène en français qu'elle ne le fait en anglais.

3. Concluons. Nous avons vu que le français et l'anglais partagent les couches morphologiques de la composition savante et de la dérivation savante. Et nous avons vu aussi que, très souvent, ces procédés de formation font usage des mêmes formes d'origine grecque et latine dans les deux langues, comme d'ailleurs dans toutes les langues de culture occidentales.

Mais nous avons découvert ensuite sous cette ressemblance superficielle deux différences fondamentales entre les deux langues.

Tout d'abord la composition savante française se trouve être la seule forme de composition de cette langue qui puisse être enchâssée dans la dérivation. Cela lui confere un rôle unique et précieux, puisque seule la composition savante se prête ainsi au développement systématique dans le domaine de la terminologie. La composition savante anglaise ne se distingue pas de la composition savante à cet égard, puisque les deux sont susceptibles d'être enchâssées dans la dérivation.

En second lieu nous avons vu que la formation des mots savants en français entretient des rapports formels très réguliers avec la formation non savante. Il y a des alternances vocaliques et consonantiques régulières qui relient entre elles formes non savantes et savantes des racines, formes finales de mot et non finales de mot d'affixes savants, et finalement certaines paires d'affixes non savants et savants. Il n'en est rien en anglais. Dans cette langue, les rapports entre lexique non savant et savant sont beaucoup moins nombreux et, pour autant qu'ils existent, beaucoup moins réguliers.

Il faut, par conséquent, se garder de conclure trop facilement au parallélisme entre français et anglais dans le domaine de la dérivation savante.

\section{ÉTUDES CITÉES}

CHOMSKY, N. et M. HALLE (1968) : The Sound Pattern of English, New York, Harper and Row.

DELL, F.C. et Elisabeth O. SELKIRK (1978) : "On a Morphologically Governed Vowel Alternation in

French ", dans S.J. Keyser (édit.) : Recent Transformational Studies in European Languages, Cambridge, Ma, MIT Press.

NYROP, Kr. (1908) : Grammaire historique de la langue française 3, Gyldendalske Boghandel-Nordisk Forlag.

PICHON, E. (1942) : les Principes de la suffixation en français, Paris, d'Artrey.

SELKIRK, Elisabeth O. (1982) : The Syntax of Words, Cambridge, Ma, MIT Press.

SIEGEL, Dorothy (1974) : Topics in English Morphology, thèse de MIT, Cambridge, Ma.

ZWANENBURG, W. (1983) : Productivité morphologique et emprunt, Amsterdam, Benjamins.

ZWANENBURG, W. (à paraître) : "La compositionalité en morphologie ". 